\title{
Homocysteine as a potential biochemical marker for depression in elderly stroke survivors
}

\author{
Michaela C. Pascoe ${ }^{1 *}$, Sheila G. Crewther ${ }^{4}$, Leeanne M. Carey ${ }^{3}$, \\ Kate Noonan ${ }^{3,4}$, David P. Crewther' and Thomas Linden ${ }^{2}$ \\ 'Brain Sciences Institute, Swinburne University, Melbourne, Australia; ${ }^{2}$ Institute of Neuroscience and Physiology, \\ University of Gothenburg, Gothenburg, Sweden; ${ }^{3}$ National Stroke Research Institute, Florey Neurosciences Institutes, \\ Melbourne, Australia; ${ }^{4}$ School of Psychological Science, La Trobe University, Melbourne, Australia
}

\section{Abstract}

Background: Elderly stroke survivors have been reported to be at risk of malnutrition and depression. Vitamin B-related metabolites such as methylmalonic acid and homocysteine have been implicated in depression.

Objective: We conducted a study exploring the relationship between homocysteine and post-stroke depression.

Design: Three methodologies were used: Observational cohort study of elderly Swedish patients $(n=149)$ 1.5 years post-stroke, assessed using Diagnostic and Statistical Manual of Mental Disorders, Montgomery Åsberg Depression Rating Scale and serum blood levels of methylmalonic acid and homocysteine.

Results: Homocysteine significantly correlated with depressive symptomatology in stroke survivors $\left(\beta=0.18^{*}\right)$. Individuals with abnormal levels of methylmalonic acid and homocysteine were almost twice more likely to show depressive symptomatology than those with normal levels (depressive symptoms $22 \%$; no depressive symptoms 12\%). Comparison of methylmalonic acid and homocysteine levels with literature data showed fewer stroke survivors had vitamin deficiency than did reference individuals (normal range $66 \%$; elevated $34 \%$ ).

Conclusions: Homocysteine is significantly associated with depressive symptomatology in elderly Swedish stroke survivors.

Keywords: depression; ischemia; nutrition; neurodegeneration; geriatric

$\mathrm{D}$ epression is particularly prevalent among stroke survivors, affecting approximately a third of individuals (1). Post-stroke depression is associated with reduced functional ability and poorer outcome (2), making the prevention and management of post-stroke depression an important area of research. Vitamin deficiencies are particularly common among stroke survivors and similar to post-stroke depression are associated with poor outcome (3).

Vitamin $\mathrm{B}_{12}$ and folate are required dietary vitamin nutrients involved in the biosynthesis of depressionrelated serotonin, dopamine and norepinephrine (4). Folic acid and vitamin $B_{12}$ are also required for the synthesis of the methionine, by methionine synthase, from the amino acid homocysteine (tHcy). Methionine cannot be synthesised from tHcy without an adequate supply of folate and vitamin $\mathrm{B}_{12}$, so vitamin deficiency results in an elevated level of tHcy.

Methylmalonyl coenzyme A is the coenzyme A linked form of methylmalonic acid (MMA). Vitamin $B_{12}$ is required for the biosynthesis of MMA coenzyme $\mathrm{A}$, to succinyl coenzyme A in the citric acid cycle. Therefore, insufficient vitamin $B_{12}$ levels are associated with increased levels of MMA (5).

High tHcy is associated with a $70 \%$ increased risk of clinical depression in elderly individuals (6), while folate is decreased in blood samples of depressed individuals (7). Low folate is associated with a worse than expected response to selective serotonin reuptake inhibitor (SSRI) and tricyclic antidepressants, while higher folate levels are 
associated with better response $(8,9)$. Supplementation with folate has been shown to have antidepressant effects and to be as effective as conventional antidepressants in some cases $(6,10,11)$. Higher tHcy contributes to depression-related naturally occurring cell death or apoptosis $(7,12)$.

Thus, as elderly stroke patients are at particular risk of malnourishment (3) and depression (1) and as B vitaminrelated factors such as folate and Vitamin $\mathrm{B}_{12}$ related to tHcy and MMA are implicated in the aetiology of clinical depression, it is particularly important to clarify if these biomarkers are associated with the depressive symptomatology seen in at least one third of elderly stroke patients (1).

We sought to systematically review the role of MMA, tHcy, folate and Vitamin $\mathrm{B}_{12}$ and depressive symptomatology in stroke survivors. To do this, we examined the vast amount of literature relating to the relationship between $\mathrm{B}$ vitamins and clinical depression, and between $\mathrm{B}$ vitamins and stroke. No studies were found to directly address the possible role of folate, vitamin $\mathrm{B}_{12}$, tHcy or MMA as contributing to depressive symptomatology in a post-stroke sample.

Thus, we aimed to explore the relationship between tHcy and depressive symptomatology among chronic stroke survivors, by determining the percentage of individuals diagnosed with clinical depression and vitamin deficiency. We further aimed to determine if elevated tHcy contributed to the observed depressive symptomatology and to clarify the percentage of elderly stroke survivors with abnormal tHcy and MMA, compared to a matched non-stroke affected population. Thus, we compared a population of elderly Swedish stroke survivors to a matched non-stroke population of elderly Swedish individuals (13) to assess nutrition-induced risk for deficiency in the depression-related metabolites MMA and tHcy, 1.5 years post-stroke.

\section{Method}

\section{Subjects}

Participant selection has been described in detail previously (14). Briefly, participants $(n=149)$ were drawn from the 'Gothenburg 70+ Stroke Study,' which consisted of individuals admitted to Sahlgrenska University Hospital, Gothenburg, Sweden, between 1 February 1993 and 17 May 1994, aged at least 70 years and presenting with an acute cerebrovascular neurological deficit, as diagnosed by routine investigations by the physician on call and acute computer tomography (CT) scan. Exclusion from the study applied if coma, cerebral tumour, extracerebral or subarachnoid haemorrhage, previous cerebral lesion requiring ongoing care, or a requirement for specialised neurological care was present. Participation was limited also to individuals who received a bed in the stroke unit, presented with symptoms for a time period no greater than 7 days prior to admission and were not residing in a nursing home at the time of admission. There was no control participant data available for this cohort study.

\section{Study protocol}

Stroke patients were contacted by mail 1 year after stroke and subsequently by phone, to arrange a hospital-based appointment. When unable to attend the hospital-based appointment $(n=15 ; 10 \%)$, interviews took place in participants' homes. Informed consent was obtained after having provided verbal and written information to participants or nearest relatives when relevant. Ethics approval was granted by The Ethics Committee for Medical Research at the University of Gothenburg.

\section{Biomarkers collection}

MMA was analysed from venous blood drawn into gel tubes (filled to at least three quarters full), turned over at least 10 times and centrifuged at $2300 \mathrm{~g}$ for $10 \mathrm{~min}$. Serum was separated and kept at room temperature before analysis with gas chromatography/mass spectrometry. Samples for tHcy were drawn in Li-heparin gel tubes (filled to at least three quarters full) turned over at least five times within $30 \mathrm{~min}$ and centrifuged at $2300 \mathrm{G}$ for $10 \mathrm{~min}$, before analysis. Reference values from the Uddevalla Hospital, Uddevalla, Sweden were $<0.37$ $\mu \mathrm{mol} / \mathrm{L}$ for serum MMA, $<15 \mu \mathrm{mol} / \mathrm{L}$ for serum tHcy (13).

\section{Diagnostic criteria}

Depression assessments were conducted by a neurologist/ psychiatrist who was unaware of the type, size and location of the index stroke at the time of the investigation and throughout the diagnostic procedure. Clinical depression was diagnosed according to DSM-III-R criteria (15) using algorithms based on psychiatric interview and neuropsychiatric examination. Depressive symptomatology was assessed using the Montgomery Åsberg Depression Rating Scale (MADRS). Adjusted MADRS scores are compiled based on information obtained from 10 related criteria, such as suicidal thoughts and pessimistic thoughts. Individuals are given a score for each of these measures that are complied into an overall depressive score. Missing data, however, on some of these criteria among cases made MADRS score adjustment implausible in the current study; therefore, raw score data were used as a conservative measure of depressive symptoms. It should be noted, however, that the raw score data used in the present study may underestimate the degree of depressive symptomatology among the sample.

Assessment consisted of a semi-structured diagnostic psychiatric interview, neuropsychological tests and a 
neurological examination. Assessment instruments were the same as those used in the Gerontological and Geriatric Population Studies and the Prospective Population Study on Women and included a semi-structured psychiatric diagnostic interview with the Comprehensive Psychopathological Rating Scale (16). This form of clinical depression diagnosis was used to categorically classify individuals as either depressed or not depressed, in order to determine the percentage of these individuals with and without elevated MMA and tHcy. For the purpose of multiple regression analysis and descriptive statistics, raw continuous MADRS scores only were used.

\section{Control reference data}

Control participant reference data were sourced from PubMed and drawn from a population-based study of elderly Swedish individuals (13). This study provided information regarding tHcy and MMA levels in nonstroke elderly Swedish individuals and laboratory cutoff points for vitamin deficiency. This control data were compared with the stroke cohort data to assess if elderly stroke survivors were more at risk of nutritional deficiencies, as indicated by MMA and tHcy 1.5 years poststroke, than were the non-stroke elderly individuals.

\section{Statistical methods}

Statistical analysis was conducted using the SPSS version 18 package. Hierarchical Multiple Regression was used to determine the variance explained by tHcy after accounting for age and gender. Outliers were screened using box plots; linearity and homoscedasticity were checked using scatter plots of standardized residuals. Normality was assessed using a histogram and normal probability plots. Pearson's Correlation was used to determine multi-collinearity between predictors. Independent sample $t$ tests were used to determine the differences in MMA and tHcy expression between stroke survivors and reference data from non-stroke individuals.

\section{Results}

Characteristics of the observational cohort study population Table 1 illustrates the characteristics of stroke survivors according to gender. The study cohort consisted of 243 patients at baseline (stroke admission). By the time of hospital follow-up, at 1.5 years post-stroke (SD, 0.4 years), 32\% $(n=77)$ had passed away and 7\% $(n=17)$ declined the invitation to participate, leaving 149 individuals. Two thirds, $65 \%(n=97)$ of individuals were female, and 35\% $(n=52)$ were male. Patients mean age at follow-up was 81 years (SD 5.3 years). The mean age of the female survivors was approximately 2 years older than that of the male participants. Female MADRS scores were approximately three points higher than male
Table 1. Age, Montgomery Åsberg Depression Rating Scale (MADRS) scores, homocysteine (tHcy) and methylmalonic acid (MMA) in stroke survivors.

\begin{tabular}{lccc}
\hline & $N$ & Mean (SE) & Median \\
\hline Female & 97 & & \\
Age & & $82(0.55)$ & 83.08 \\
MADRS & & $11.40(0.90)$ & 9 \\
tHcy & 79 & $12.24(0.64)$ & 11 \\
MMA & 78 & $0.27(0.01)$ & 0.25 \\
Male & 52 & & \\
Age & & $79.23(0.64)$ & 78.99 \\
MADRS & & $8.23(0.90)$ & 7 \\
tHcy & 39 & $12.05(0.69)$ & 11 \\
MMA & 38 & $0.24(0.01)$ & 0.23 \\
\hline
\end{tabular}

$>15 \mu \mathrm{mol} / \mathrm{L}=$ cut off point of thcy. $>0.37 \mu \mathrm{mol} / \mathrm{L}=$ cut off point of serum MMA. Higher MADRS scores represent more depressive symptomatology. Means, Medians and SE Including Age, Gender, tHcy Expression and MADRS Scores, Presented by Gender.

participants. tHcy was comparable between males and females.

\section{Rates of depression and vitamin deficiency among stroke survivors}

Of the individuals with elevated metabolites, $22 \%$ showed depressive symptoms, while $12 \%$ showed no depressive symptoms. Of the individuals with no elevated metabolites, only $16 \%$ showed depressive symptoms, while $37 \%$ showed no depressive symptoms. Individuals with elevated tHcy or MMA were almost twice as likely to be classified as clinically depressed, according to Diagnostic and Statistical Manual of Mental Disorders (DSM-III) criteria, than were individuals who did not show elevated tHcy or MMA.

As expected, MMA and tHcy were significantly correlated ( $r=0.17, n=115, p=0.03)$.

\section{Multiple regression}

Table 2 shows the results of the Hierarchical Multiple Regression used to determine the predictive roles of age, gender and peripheral blood plasma tHcy expression on raw MADRS scores. Age and gender were entered together into the first block, as previous research with this particular cohort indicated that both contributed to depressive symptoms (1). Then, tHcy was entered into the second block to determine if it contributed to the MADRS scores. Age and gender together explained $3.2 \%$ of the variance in depression scores, with neither gender nor age predicting depression scores $(F(2,115)=$ $1.88, p=0.16)$. The addition of thcy to the model increased the amount of variance explained by $3.3 \%$, with tHcy significantly contributing to MADRS scores $(F(1,114)=3.99, p<0.05)$. Table 2 displays the 
Table 2. Coefficients, standard error of the coefficients and standardised beta values of the regression model.

\begin{tabular}{lrrrr}
\hline & B & SE B & $\beta$ & R2 \\
\hline Step I & & & & 0.03 \\
$\quad$ Constant & 12.42 & 12.42 & & \\
Age & -0.13 & 0.15 & -0.08 & \\
Gender & -3.08 & 1.63 & -0.18 & \\
Step 2 & & & & 0.06 \\
Constant & 12.08 & 12.26 & & \\
Age & -0.05 & 0.15 & -0.03 & \\
Gender & -3.13 & 1.61 & -0.18 & \\
tHcy & 0.28 & 0.14 & $0.18^{*}$ & \\
\hline
\end{tabular}

Note: $R 2=0.03$ for Step I: $\Delta R 2=0.06$ for Step $2(p<0.05)$.

*Significant at $p<0.05$.

coefficients $(b)$ the Standard Error of the coefficients (SE B) and standardised beta ( $\beta$ ) values of the model.

\section{Characteristics of the study population as compared to literature sourced control data}

As no control data were available for the observation cohort study, reference values of tHcy and MMA expression in elderly non-stroke-affected Swedish individuals was sourced from the literature (13). One sample $t$ tests were used to show that stroke survivors differed significantly in MMA $t(147)=-3.528$, $p<0.001$ (Stroke $=0.20$; Control $=0.16)$ and tHcy levels, $t(148)=-9.64, p<0.001($ Stroke $=9.64$; Control $=14.7)$. Surprisingly, $49 \%$ of non-stroke individuals showed elevated tHcy and MMA. Among stroke survivors, 34\% of individuals had elevated tHcy or and MMA as compared to the literature-sourced reference values (13).

\section{Discussion}

To our knowledge this is the first observational cohort study to examine the relationship between depressive symptoms and tHcy, a sensitive measure of folate and vitamin $B_{12}$ status (17), in an at-risk elderly post-stroke population.

Individuals with elevated tHcy or MMA were almost twice more likely to be classified as clinically depressed according to DSM-III criteria than were individuals who did not show elevated tHcy or MMA. The multiple regression showed that peripheral plasma tHcy was significantly associated with MADRS depression scores after having controlled for age and gender. This suggests that tHcy is a predictor of depressive symptomatology in stroke survivors. These findings are consistent with previous research suggesting that in non-stroke individuals, increased folate and vitamin $\mathrm{B}_{12}$ may decrease depressive symptoms by influencing the production of depression-related neurotransmitters (4).
Recently, research has begun to focus also on the role of apoptosis in stroke-related depression (18). Apoptosis is elevated among depressed individuals (14) and considered to be a risk factor for depressive disorder (19). The metabolite tHcy is toxic to neurons (20). Thus, tHcy may be contributing to depressive symptomatology, in part, via regulation of depression-related apoptosis.

In the present study, no gender differences were found in tHcy expression. Females did, however, have higher MADRS scores than males. This finding is consistent with previous analysis using data from this cohort (1) and the general trend of higher reported levels of subjective depression among females (21).

The present research additionally aimed to explore if stroke survivors were more at risk of vitamin deficiency, as indicated by MMA and tHcy levels, than was a comparable non stroke affected population. Previous research has indicated that age-related biological and physiological changes make the elderly particularly vulnerable to vitamin deficiency (22). Dysphagia and subsequent eating difficulties in up to $80 \%$ of patients with acute stroke has been reported to further increase this risk of malnutrition (3). Stroke survivors have additionally been shown to be particularly at risk of clinical depression (1); thus, in the present study it was important to clarify the presence of nutritional deficiencies among stroke survivors in depression-associated markers. In this cohort, Swedish stroke survivors were no more likely to show elevated MMA or tHcy, 1.5 years after a stroke, compared to elderly Swedish nonstroke-affected individuals. In fact, among stroke survivors, a smaller percentage of individuals were found to be vitamin deficient than in the reference group. However, caution should be exercised when interpreting these results. In the present study, nearly one third of stroke survivors were found to be vitamin deficient as indicated by elevated tHcy and MMA, and thus should still be considered an 'at risk' group. This is particularly true as tHcy elevation was associated with depressive symptomatology in the stroke surviving cohort. Elderly individuals are in general identified as at risk of nutritional deficiencies, due to an extensive variety of factors spanning from psychosocial, social, financial, medical and cognitive (22). Very high levels of nutritional deficiency among the control group may have overshadowed the observed deficiency among strokesurviving individuals. Indeed, almost half of the elderly Swedes from the reference study had elevated MMA and tHcy (21). It may also be the case that these particular stroke survivors showed less vitamin deficiency than the non-stroke-affected comparison population due to the typically high level of medical care, likely hospitalisation and monitoring given post-stroke in Sweden. Finally, the stroke experience may prompt increased self awareness regarding health and well-being, 
which in conjunction with high-level medical care may have encouraged stroke survivors to consume more nutritionally sound foods.

\section{Conclusions}

The present observational cohort study demonstrates that MMA and tHcy are associated with a depression diagnosis post-stroke, and that high tHcy expression is a significant predictor of depressive symptoms post-stroke after accounting for age and gender. The results are consistent with the argument that nutritional factors post-stroke are important in understanding depressive disorder and its prevention. This observational cohort data additionally demonstrates that elderly Swedish stroke survivors do not have more B vitamin deficiencies when compared to elderly non-stroke-affected Swedish individuals. It is important to note, however, that this data were collected in the 1990s and possible changes in nutritional intake since this time among stroke survivors may influence the present day validity of these findings. While we do not anticipate this to be the case, we do suggest that replicating the present study to ensure the validity of the results is recommended.

\section{Statement of Authorship}

The authors' responsibilities were as follows - TL: Contributed to study design, TL: Contributed to data collection; MCP, TL, SGC, LMC: contributed to the conception and design of the manuscript, $\mathrm{MCP}, \mathrm{KN}$ : contributed to the literature search, MCP: Analysed and interpreted data; MCP: wrote the manuscript; and SGC, LMC, DPC, TL: critically appraised the manuscript. All authors have made substantial contributions and final approval of the conceptions, drafting and final version.

\section{Acknowledgements}

Special thanks to Dr Sean Cowlishaw for assisting with the data analysis. Special thanks to the Institute of Neuroscience and Physiology, University of Gothenburg for assistance with the data collection.

\section{Conflict of interest and funding}

The authors declare no conflict of interest.

\section{References}

1. Linden T, Blomstrand C, Skoog I. Depressive disorders after 20 months in elderly stroke patients: a case-control study. Stroke 2007; 38: 1860-3.

2. Masskulpan P, Riewthong K, Dajpratham P, Kuptniratsaikul V. Anxiety and depressive symptoms after stroke in 9 rehabilitation centers. J Med Assoc Thai 2008; 91: 1595-602.

3. Ha L, Iversen PO, Hauge T. Nutrition for elderly acute stroke patients. Tidsskr Nor Laegeforen 2008; 128: 1946-50.

4. Hutto BR. Folate and cobalamin in psychiatric illness. Compr Psychiatry 1997; 38: 305-14.
5. Klee GG. Cobalamin and folate evaluation: measurement of methylmalonic acid and homocysteine vs vitamin $\mathrm{B}(12)$ and folate. Clin Chem 2000; 46: 1277-83.

6. Almeida OP, McCaul K, Hankey GJ, Norman P, Jamrozik K, Flicker L. Homocysteine and depression in later life. Arch Gen Psychiatry 2008; 65: 1286-94.

7. Gilbody S, Lightfoot T, Sheldon T. Is low folate a risk factor for depression? A meta-analysis and exploration of heterogeneity. J Epidemiol Community Health 2007; 61: 631-7.

8. Alpert M, Silva RR, Pouget ER. Prediction of treatment response in geriatric depression from baseline folate level: interaction with an SSRI or a tricyclic antidepressant. J Clin Psychopharmacol 2003; 23: 309-13.

9. Taylor MJ, Carney SM, Goodwin GM, Geddes JR. Folate for depressive disorders: systematic review and meta-analysis of randomized controlled trials. J Psychopharmacol 2004; 18: 251-6.

10. Jorm AF, Christensen H, Griffiths KM, Rodgers B. Effectiveness of complementary and self-help treatments for depression. Med J Aust 2002; 176: 84S-96S.

11. Frazer CJ, Christensen H, Griffiths KM. Effectiveness of treatments for depression in older people. Med J Aust 2005; 182: 627-32.

12. Fava M, Borus JS, Alpert JE, Nierenberg AA, Rosenbaum JF, Bottiglieri T. Folate, vitamin B12, and homocysteine in major depressive disorder. Am J Psychiatry 1997; 154: 426-8.

13. Bjorkegren K, Svardsudd K. Serum cobalamin, folate, methylmalonic acid and total homocysteine as vitamin B12 and folate tissue deficiency markers amongst elderly Swedes - a population-based study. J Intern Med 2001; 249: 423-32.

14. Eilat E, Mendlovic S, Doron A, Zakuth V, Spirer Z. Increased apoptosis in patients with major depression: a preliminary study. J Immunol 1999; 163: 533-4.

15. Association AP. DSM-III-R. Diagnostic and statistical manual of mental disorders. Washington, D.C: Amer Psychiatric Pub; 1987.

16. Asberg M, Montgomery SA, Perris C, Schalling D, Sedvall G. A comprehensive psychopathological rating scale. Acta Psychiatr Scand Suppl 1978; 271: 5S-27S.

17. Selhub J, Jacques PF, Dallal G, Choumenkovitch S, Rogers G. The use of blood concentrations of vitamins and their respective functional indicators to define folate and vitamin B12 status. Food Nutr Bull 2008; 29: 67S-73S.

18. Pascoe MC, Crewther SG, Carey LM, Crewther DP. What you eat is what you are - a role for polyunsaturated fatty acids in neuroinflammation induced depression? Clin Nutr 2011; 30: $407-15$.

19. Harlan J, Chen Y, Gubbins E, Mueller R, Roch JM, Walter K, et al. Variants in Apaf-1 segregating with major depression promote apoptosome function. Mol Psychiatry 2006; 11: 76-85.

20. Loscalzo J. The oxidant stress of hyperhomocyst(e)inemia. J Clin Invest 1996; 98: 5-7.

21. Kuehner C. Gender differences in unipolar depression: an update of epidemiological findings and possible explanations. Acta Psychiatr Scand 2003; 108: 163-74.

22. Brownie S. Why are elderly individuals at risk of nutritional deficiency? Int J Nurs Pract 2006; 12: 110-8.

\footnotetext{
* Michaela Pascoe

Brain Sciences Institute

Life and Social Sciences

Swinburne University

Melbourne 3086

Australia

Tel: +61 390357107

Fax: +61392145525

Email: mpascoe@swin.edu.au
} 